$54^{\text {ème }}$ Congrès de la SFMBCB, 03007 (2011)

DOI: $10.1051 / \mathrm{sfmbcb} / 20115403007$

(C) Owned by the authors, published by EDP Sciences, 2011

\title{
Manifestations parodontales des malformations artério-veineuses : difficultés diagnostiques et thérapeutiques
}

\author{
Ella B, Meyer M, Lanotte A, Fricain JC \\ Départements de Parodontologie et de Pathologie buccale, UFR d'Odontologie, Bordeaux, France \\ tsir.ella@wanadoo.fr
}

Les malformations artério-veineuses (MAV) constituent les malformations vasculaires superficielles les plus fréquentes. Ce sont des malformations congénitales des vaisseaux sanguins. Elles sont toujours présentes à la naissance mais peuvent passer inaperçues pendant quelques temps. Dans de rares cas, elles ne se manifestent qu'à l'âge adulte (El Kohen 2003). Elles se caractérisent par l'existence d'une communication anormale, entre les artères et les veines, avec hémodétournement. Le lit capillaire est remplacé par d'importantes « tortuosités » vasculaires et par des zones de communication anormales directes entre artères et veines (shunts). Elles sont hémodynamiquement actives et représentent un véritable défi pour les praticiens lorsqu'il existe un flux sanguin à haut débit et de multiples collatérales (Kademani 2004, Seemann 2005). Les MAV de la région maxillo-faciale sont rares (Rodesch 1998).

Nous décrivons un cas clinique de MAV maxillaire qui a été initialement traité comme une parodontite. Il s'agit d'une femme de 44 ans, suivie par son dentiste depuis 1996 pour une parodontite de l'adulte avec un foyer réfractaire dans le secteur prémolo-molaire droit. La patiente signalait déjà l'existence d'une petite lésion très hémorragique en regard de 15,16 et 17. Malgré un traitement parodontal soutenu, la 16 a dû être extraite en 1999. En 2004, la patiente a été adressée dans le département de Parodontologie et de Pathologie buccale du CHU de Bordeaux. Elle présentait une lésion hémorragique volumineuse en regard, de 14 et 15 . Des examens complémentaires ont été réalisés, parmi lesquels un examen anatomopathologique et une échotomographie vasculaire associée à un doppler pulsé qui ont révélé une MAV maxillaire sans shunt. Ces dents ont été extraites en 2005 avec ablation de la lésion hémorragique. La rémission obtenue par corticothérapie avait permis d'envisager une réhabilitation implanto-portée du secteur édenté.

Le diagnostic des MAV associées à des manifestations parodontales est parfois difficile à évoquer. Leur pronostic est réservé et quelquefois elles mettent en jeu le pronostic vital. Cliniquement elles peuvent prendre l'aspect d'une épulis, d'une gingivite érosive, d'une parodontite ou d'un carcinome épidermoïde bourgeonnant de la gencive (Samson et al. 1997, Gauzeran 2004). La prise en charge de cette pathologie doit être pluridisciplinaire. Le doppler pulsé et l'artériographie sont des examens clés pour l'exploration des MAV superficielles (Gold 2003). Le traitement chirurgical est indiqué en cas d'évolutivité importante mais, le plus souvent, il vise a obtenir une restauration fonctionnelle et esthétique (Baud 2000, Giaoui 2003). 